PROCEEDINGS OF THE

AMERICAN MATHEMATICAL SOCIETY

Volume 127, Number 1, January 1999, Pages 95-103

S 0002-9939(99)04906-0

\title{
HAMMERSTEIN INTEGRAL INCLUSIONS IN REFLEXIVE BANACH SPACES
}

\author{
TIZIANA CARDINALI AND NIKOLAOS S. PAPAGEORGIOU
}

(Communicated by Palle E. T. Jorgensen)

\begin{abstract}
In this paper we examine multivalued Hammerstein integral equations defined in a separable reflexive Banach space. We prove existence theorems for both the "convex" problem (the multifunction is convex-valued) and the "nonconvex" problem (the multifunction is not necessarily convex-valued). We also show that the solution set of the latter is dense in the solution set of the former (relaxation theorem). Finally we present some examples illustrating the applicability of our abstract results.
\end{abstract}

\section{INTRODUCTION}

Integral equations of the Hammerstein type have been studied by many authors and have been one of the most important domains of application of the ideas and methods of nonlinear functional analysis and in particular of the theory of nonlinear operators of monotone type. We refer to the works of Browder [6], Amann [2], Brezis-Browder [5], Ahmed [1], O'Regan [13] and the references therein.

Various applied problems arising in mathematical physics, mechanics and control theory lead to multivalued analogs of the Hammerstein integral equations, the socalled "Hammerstein integral inclusions". In this direction we have the works of Lyapin [11], Coffman [8], Glashoff-Sperkels [9], Papageorgiou [18], Appell et al. [3] and O'Regan [14]. Most of the existence theorems proved in the above works are based on the fixed point principles of Nadler and of Kakutani-KyFan (see KleinThompson [10]). These fixed point principles are multivalued analogs of the Banach and Schauder-Tichonov fixed point theorems respectively. Only Coffman [8], Appell et al. [3] and O'Regan [14] used different approaches. Coffman studied eigenvalue problems by means of a topological characteristic (called "genus") for set-valued operators. Appell et al. [3] and O'Regan [14] used multivalued variants of the Leray-Schauder principle. The first of these works used the theory of ideal spaces, while the second deals with superlinear integral inclusions defined on $\mathbb{R}$.

In this paper we consider integral inclusions in reflexive Banach spaces. Our main theoretical tool is the Leray-Schauder alternative theorem and our approach does not use ideal spaces. So our results extend in many respects those of Appell et al. and O'Regan. In particular the multifunction $F$ is Banach space-valued

Received by the editors March 6, 1997.

1991 Mathematics Subject Classification. Primary 47H04, 47H30, 45G10.

Key words and phrases. Compact operator, measurable multifunction, lsc and usc multifunction, multivalued Nemitsky operator, $h$-continuous multifunction, Leray-Schauder alternative theorem, relaxation theorem, elliptic inclusions, Green's operator. 
(Appell et al. have $X=\mathbb{R}^{N}$, while O'Regan has $X=\mathbb{R}$ ) and our hypotheses on the data are in general easier to verify in concrete situations. In contrast to Appell et al., we also deal with the nonconvex problem (this is done also by O'Regan) and we prove a relaxation theorem, which is missing from both Appell and O'Regan. Moreover in Theorem 7 we do not require the "integral" operator to be linear. In the last section of the paper we have gathered some characteristic applications of our abstract results.

\section{Preliminaries}

Let $(Z, \Sigma, \mu)$ be a measure space and $X$ a separable Banach space, with $X^{*}$ its topological dual. We will be using the following notation: $P_{f(c)}(X)=\{A \subseteq X: A$ is nonempty, closed (and convex) $\}$ and $P_{k(c)}(X)=\{A \subseteq X: A$ is nonempty, compact (and convex)\}. For $A \in 2^{X} \backslash\{\varnothing\}$ we set $|A|=\sup [\|x\|: x \in A]$. A multifunction $F: Z \rightarrow P_{f}(X)$ is said to be "measurable", if for all $U \subseteq X$ open, $F^{-}(U)=\{z \in$ $Z: F(z) \cap U \neq \varnothing\} \in \Sigma$. If in addition we assume that $\mu$ is a complete, $\sigma$-finite measure defined on $\Sigma$, then the above definition of measurability is equivalent to saying that $G r F=\{(z, x) \in Z \times X: x \in F(z)\} \in \Sigma \times B(X)$, where $B(X)$ is the Borel $\sigma$-field of $X$ (graph measurability). If $F: Z \rightarrow P_{f}(X)$ is a measurable multifunction, then for $1 \leq p \leq \infty$ we define the set $S_{F}^{p}=\left\{f \in L^{p}(Z, X): f(z) \in F(z) \mu\right.$-a.e. $\}$, i.e. $S_{F}^{p}$ contains all selectors of $F(\cdot)$ which belong to the Lebesgue-Bochner space $L^{p}(Z, X)$.

If $A, C \in 2^{X} \backslash\{\varnothing\}$, then we define the "Hausdorff distance" of $A$ and $C$ to be the number $h(A, C)=\max \left[\sup _{a \in A} d(a, C), \sup _{c \in C} d(c, A)\right]$, where $d(a, C)=$ $\inf [\|a-c\|: c \in C]$ and $d(c, A)=\inf [\|c-a\|: a \in A]$. A multifunction $F: X \rightarrow$ $P_{f}(X)$ is said to be " $h$-continuous", if it is continuous into the metric space $\left(P_{f}(X), h\right)$.

Finally if $V, Y$ are Hausdorff topological spaces, a multifunction $F: V \rightarrow 2^{Y} \backslash\{\varnothing\}$ is said to be upper semicontinuous (usc) (resp. lower semicontinuous (lsc)), if, for any $C \subseteq Y$ closed, $F^{-}(C)=\{v \in V: F(v) \cap C \neq \varnothing\}$ (resp. $F^{+}(C)=\{v \in$ $V: F(v) \subseteq C\})$ is closed in $V$.

\section{Existence theorems}

Let $(Z, \Sigma, \mu)$ be a finite measure space and $X$ a separable reflexive Banach space. Given $w \in L^{p}(Z, X)(1<p<\infty)$, we consider the following abstract multivalued Hammerstein equation (Hammerstein inclusion):

$$
y \in K N(y)+w .
$$

Here $N: L^{p}(Z, X) \rightarrow 2^{L^{q}\left(Z, X^{*}\right)}$ is the multivalued Nemitsky operator corresponding to the multifunction $F: Z \times X \rightarrow 2^{X^{*}} \backslash\{\varnothing\}$ (i.e. $N(y)=\left\{u \in L^{q}\left(Z, X^{*}\right)\right.$ : $u(z) \in F(z, y(z)) \mu$-a.e. on $\left.Z\}=S_{F(\cdot, y(\cdot))}^{q}\right)$ and $K: L^{q}\left(Z, X^{*}\right) \rightarrow L^{p}(Z, X)$ is a compact linear operator (i.e. $K(\cdot)$ is continuous and maps bounded sets to relatively compact sets). Here $1<p<\infty, \frac{1}{p}+\frac{1}{q}=1$ and recall that $L^{p}(Z, X)^{*}=L^{q}\left(Z, X^{*}\right)$.

First we determine the properties of the multivalued Nemitsky operator.

$\mathbf{H}(\mathbf{F})_{1} . F: Z \times X \rightarrow P_{f c}\left(X^{*}\right)$ is a multifunction such that (i) for all $y \in X$, $z \rightarrow F(z, y)$ is measurable; (ii) for $\mu$-almost all $z \in Z, \operatorname{Gr} F(z, \cdot)$ is sequentially closed in $X \times X_{w}^{*}$, where $X_{w}^{*}$ denotes the space $X^{*}$ furnished with the weak topology; (iii) for almost all $z \in Z$ and all $y \in X,|F(z, y)| \leq a(z)+c\|y\|^{p-1}$, where $a \in L^{q}(Z)$, $c>0$. 
Proposition 1. If hypotheses $H(F)_{1}$ hold,

then $N: L^{p}(Z, X) \rightarrow P_{f c}\left(L^{q}\left(Z, X^{*}\right)\right)$ is usc into $L^{q}\left(Z, X^{*}\right)$ equipped with the weak topology.

Proof. Let $C \subseteq L^{q}\left(Z, X^{*}\right)$ be nonempty and weakly closed. We need to show that the set $N^{-}(C)=\left\{y \in L^{p}(Z, X): N(y) \cap C \neq \varnothing\right\}$ is closed in $L^{p}(Z, X)$ with the strong topology. To this end let $\left\{y_{n}\right\}_{n>1} \subseteq N^{-}(C)$ and assume that $y_{n} \rightarrow y$ in $L^{p}(Z, X)$. Let $u_{n} \in N\left(y_{n}\right) \cap C, n \geq 1$. From hypothesis $H(F)_{1}$ (iii) it follows that $\left\{u_{n}\right\}_{n \geq 1}$ is bounded in $L^{q}\left(Z, X^{*}\right)$. So by passing to a subsequence if necessary, we may assume that $u_{n} \stackrel{w}{\rightarrow} u$ in $L^{q}\left(Z, X^{*}\right)$. Using Theorem 3.1 of Papageorgiou [15], we have that $u(z) \in \overline{\operatorname{conv}} w-\overline{\lim } F\left(z, y_{n}(z)\right) \subseteq F(z, y(z)) \mu$-a.e. on $Z$ (the last inclusion being a consequence of hypothesis $H(F)_{1}$ (ii)). So $u \in N(y) \cap C$, hence $y \in N^{-}(C)$. Therefore $N^{-}(C)$ is closed and this completes the proof.

Now we consider the following hypotheses on $F$.

$\mathbf{H}(\mathbf{F})_{\mathbf{2}} . F: Z \times X \rightarrow P_{f c}\left(X^{*}\right)$ is a multifunction satisfying hypotheses $H(F)_{1}$ and in addition (iv) there exist $R>0$ and $c>0$ such that for $\mu$-almost all $z \in Z$, all $\|y\| \geq R$ and all $v \in F(z, y)$, we have $c\|v\|_{*}\|y\| \leq(-v, y)$ (here $\|\cdot\|_{*}$ is the norm of $X^{*}$ and $(\cdot, \cdot)$ the duality brackets for $\left.\left(X, X^{*}\right)\right)$.

Under these hypotheses, we obtain without any changes in the proof the following version of Lemma 3 of Brezis-Browder [5]. In what follows by $((\cdot, \cdot))$ we denote the duality brackets for the pair $\left(L^{p}(Z, X), L^{q}\left(Z, X^{*}\right)\right)$; i.e. $((u, y))=\int_{Z}(u(z), y(z)) d \mu(z)$ for all $u \in L^{q}\left(Z, X^{*}\right), y \in L^{p}(Z, X)$.

Proposition 2. If hypotheses $H(F)_{2}$ hold,

then there exist $c_{1}>0$ and $c_{2} \geq 0$ such that for all $y \in L^{p}(Z, X)$ and all $u \in N(y)$ we have $((-u, y)) \geq c_{1}\|u\|_{q}^{q}-c_{2}$; so for all $\eta>0$ there exists $c_{3}(\eta)>0$ such that $((-u, y)) \geq \eta\|u\|_{q}-c_{3}(\eta)$.

Let us also recall the multivalued version of the Leray-Schauder alternative theorem (see Appell et al. [3], Theorem 1).

Proposition 3. Let $E$ be a convex subset of a Banach space $V, 0 \in E$, and let $\Gamma: E \rightarrow P_{k c}(E)$ be a compact multifunction (i.e. $\Gamma(\cdot)$ is usc and maps bounded sets in $E$ into relatively compact sets). Then either (a) $\Gamma$ has a fixed point in $E$; or (b) the set $\{x \in E$ : there exists $\lambda \in(0,1)$ with $x \in \lambda \Gamma(x)\}$ is unbounded.

Our hypotheses on the operator $K(\cdot)$ are the following:

$\mathbf{H}(\mathbf{K})_{1} \cdot K: L^{q}\left(Z, X^{*}\right) \rightarrow L^{p}(Z, X)$ is a compact, linear operator and there exist $\beta>0$ and $1<\gamma<q$ such that $-\beta\|u\|_{q}^{\gamma} \leq((u, K u))$ for all $u \in L^{q}\left(Z, X^{*}\right)$.

Now we are ready for our first existence result.

Theorem 4. If hypotheses $H(F)_{2}, H(K)_{1}$ hold and $w \in L^{p}(Z, X)$,

then the solution set of problem (1) is nonempty and compact in $L^{p}(Z, X)$.

Proof. By translating things if necessary, we may assume without any loss of generality that $w=0$. Let $y \in D=\left\{y \in L^{p}(Z, X): y \in \lambda K N(y)\right.$ for some $\left.0<\lambda<1\right\}$. Then $y=\lambda K u$ with $u \in N(y)$ and $((u, y))=\lambda((u, K u))$. Using hypothesis $H(K)_{1}$ and Proposition 2, we obtain $c_{1}\|u\|_{q}^{q}-\lambda \beta\|u\|_{q}^{\gamma} \leq c_{2}$. Since $1<\gamma<q$, from the last inequality we infer that there exists $M_{1}>0$ independent of $\lambda \in(0,1)$ such that 
$\|u\|_{q} \leq M_{1}$. So we can find $M_{2}>0$ such that $\|y\|_{p}=\lambda\|K u\|_{p} \leq M_{2}$ for all $y \in D$. Let $\Gamma(y)=K N(y)$ for all $y \in L^{p}(Z, X)$. Evidently $\Gamma: L^{p}(Z, X) \rightarrow P_{k c}\left(L^{p}(Z, X)\right)$ is a compact multifunction (see Proposition 1 and hypothesis $\left.H(K)_{1}\right)$. Thus we can apply the multivalued version of the Leray-Schauder alternative theorem (see Proposition 3) and obtain $y \in L^{p}(Z, X)$ such that $y \in K N(y)$.

Next let $S$ denote the set of solutions of (1). Let $B=\left\{u \in L^{q}\left(Z, X^{*}\right)\right.$ : there exists $y \in S$ such that $y=K u\}$. From the previous proof it is clear that $B$ is bounded and so $\overline{K(B)}$ is compact in $L^{p}(Z, X)$. So it suffices to show that $S$ is closed. To this end let $\left\{y_{n}\right\}_{n \geq 1} \subseteq S$ and assume that $y_{n} \rightarrow y$ in $L^{p}(Z, X)$. Then $y_{n}=K u_{n}, u_{n} \in N\left(y_{n}\right), n \geq 1$. By virtue of hypothesis $H(F)_{1}$ (iii) and by passing to a subsequence if necessary, we may assume that $u_{n} \stackrel{w}{\rightarrow} u$ in $L^{q}\left(Z, X^{*}\right)$. As in the proof of Proposition 1 we have $u \in N(y)$ and by hypothesis $H(K)_{1}, y=K u$. So $y \in S$ and this proves the compactness of $S$ in $L^{p}(Z, X)$.

In the next existence theorem, we no longer need the hypothesis that $\mu(Z)<$ $+\infty$, but we need to impose a stronger coercivity condition on the multifunction $F$.

$\mathbf{H}(\mathbf{F})_{3} . F: Z \times X \rightarrow P_{f_{c}}\left(X^{*}\right)$ is a multifunction satisfying hypotheses $H(F)_{1}$ and (iv) there exist $c_{0}>0$ and $\theta \in L^{1}(Z)$ such that for $\mu$-almost all $z \in Z$, all $y \in X$ and all $u \in F(z, y),(-u, y) \geq c_{0}\|y\|^{p}-\theta(z)$.

Theorem 5. If $\mu(Z)$ is not necessarily finite, $H(F)_{3}, H(K)_{1}$ hold and $w \in$ $L^{p}(Z, X)$,

then problem (1) has a solution $y \in L^{p}(Z, X)$.

Proof. Again we may assume that $w=0$. Let $y \in D=\left\{y \in L^{p}(Z, X): y \in\right.$ $\lambda K N(y)$ for some $\lambda \in(0,1)\}$. Then $y=\lambda K u$ with $u \in N(y)$ and so

$$
((u, y))=\lambda((u, K u)) \geq-\lambda \beta\|u\|_{q}^{\gamma} .
$$

Also by virtue of hypothesis $H(F)_{3}$ (iv) we have

$$
((u, y)) \leq\|\theta\|_{1}-c_{0}\|y\|_{p}^{p}
$$

Moreover from the growth condition on $F$ (see $H(F)_{1}$ (iii)), we obtain $\|u\|_{q} \leq$ $\|a\|_{q}+c\|y\|_{q}^{p-1}$ and so we deduce that

$$
\|u\|_{q}^{\gamma} \leq 2^{\gamma-1}\|a\|_{q}^{\gamma}+2^{\gamma-1} c^{\gamma}\|y\|_{p}^{\gamma(p-1)} .
$$

Using inequalities (3) and (4) in (2), we obtain

$$
c_{0}\|y\|_{p}^{p}-\lambda 2^{\gamma-1} c^{\gamma}\|y\|_{p}^{\gamma(p-1)} \leq c_{3}
$$

for some $c_{3}>0$ independent of $\lambda \in(0,1)$. Since $\gamma(p-1)<p$, from (5) above it follows that there exists $M>0$ independent of $\lambda \in(0,1)$ such that $\|y\|_{p} \leq M$ for all $y \in D$. Invoking Proposition 3 , we conclude that there exists $y \in L^{p}(Z, X)$ such that $y \in K N(y)$.

Now we pass to the "nonconvex" problem (i.e. the multivalued term $F(z, y)$ need not have convex values). The precise hypotheses on $F$ are:

$\mathbf{H}(\mathbf{F})_{4} \cdot F: Z \times X \rightarrow P_{f}\left(X^{*}\right)$ is a multifunction such that (i) $F(\cdot, \cdot)$ is superpositionally graph measurable (i.e. if $x: Z \rightarrow X$ is measurable, then $z \rightarrow F(z, x(z))$ is graph measurable); (ii) for almost all $z \in Z, y \rightarrow F(z, y)$ is lsc; (iii) for $\mu$-almost all $z \in Z$ and all $y \in X,|F(z, y)| \leq a(z)+c\|y\|^{p-1}$ where $a \in L^{q}(Z), c \geq 0$; (iv) either $H(F)_{2}$ (iv) or $H(F)_{3}$ (iv) (with $\mu(z) \leq \infty$ ) holds. 
Proposition 6. If hypotheses $H(F)_{4}$ (i), (ii) and (iii) hold,

then $N: L^{p}(Z, X) \rightarrow P_{f}\left(L^{q}\left(Z, X^{*}\right)\right)$ is lsc.

Proof. We need to show that for every nonempty closed set $C \subseteq L^{q}\left(Z, X^{*}\right), N^{+}(C)$ is closed. To this end let $\left\{y_{n}\right\}_{n \geq 1} \subseteq N^{+}(C)$ and assume that $y_{n} \rightarrow y$ in $L^{p}(Z, X)$. By passing to a subsequence if necessary, we may assume that $y_{n}(z) \rightarrow y(z)$ a.e. on $Z$. Let $u \in N(y)$. For every $n \geq 1$ through a straightforward application of the $\mathrm{Au}-$ mann selection theorem (see Klein-Thompson [10]), we can produce $u_{n} \in L^{q}\left(Z, X^{*}\right)$ such that $u_{n}(z) \in F\left(z, y_{n}(z)\right) \mu$-a.e. on $Z$ and such that $\left\|u(z)-u_{n}(z)\right\|_{*} \leq$ $d\left(u(z), F\left(z, y_{n}(z)\right)\right)+\frac{1}{n}$ a.e. on $Z$. Hence

$$
\varlimsup\left\|u(z)-u_{n}(z)\right\| \leq \varlimsup \lim d\left(u(z), F\left(z, y_{n}(z)\right)\right) \quad \mu \text {-a.e. on } Z .
$$

But since by hypothesis $H(F)_{4}$ (ii) for $\mu$-almost all $z \in Z, y \rightarrow F(z, y)$ is lsc, the function $y \rightarrow d(u(z), F(z, y))$ is upper semicontinuous and so $\varlimsup$ iim $d\left(u(z), F\left(z, y_{n}(z)\right)\right)$ $\leq d(u(z), F(z, y(z)))=0 \mu$-a.e. on $Z$. Therefore $u_{n}(z) \rightarrow u(z) \mu$-a.e. in $X$. Thus by the dominated convergence theorem $u_{n} \rightarrow u$ in $L^{q}\left(Z, X^{*}\right)$. But $u_{n} \in N\left(y_{n}\right) \subseteq C$ and so $u \in C$. Therefore $N^{+}(C)$ is closed and this completes the proof of the proposition.

Using this proposition, we can prove the following existence theorem.

Theorem 7. If $H(K)_{1}$ with $K$ not necessarily linear and $H(F)_{4}$ hold and $w \in$ $L^{p}(Z, X)$,

then problem (1) has a solution $y \in L^{p}(Z, X)$.

Proof. By Proposition 6 the Nemitsky operator $N: L^{p}(Z, X) \rightarrow P_{f}\left(L^{q}\left(Z, X^{*}\right)\right)$ is lsc and has decomposable values (i.e. if $\left(u_{1}, u_{2}, A\right) \in N(y) \times N(y) \times \Sigma$, then $\left.\chi_{A} u_{1}+\chi_{A^{c}} u_{2} \in N(y)\right)$. So we can apply Theorem 3 of Bressan-Colombo [4] and obtain a continuous map $h: L^{p}(Z, X) \rightarrow L^{q}\left(Z, X^{*}\right)$ such that $h(y) \in N(y)$ for all $y \in L^{p}(Z, X)$. Then consider the abstract Hammerstein equation $y=K h(y)+w$. Proceed as in the proof of Theorem 1 or Theorem 2, to obtain a solution $y \in$ $L^{p}(Z, X)$. Note that the single-valuedness of the problem permits us to drop the linearity requirement on $K$.

\section{Relaxation theorem}

In this section we prove a relaxation theorem, namely we show that the solutions of the nonconvex problem are dense in $L^{p}(Z, X)$ in the solutions of the convex problem. So we consider

$$
y \in K N(y) \quad\left(N(y)=S_{F(\cdot, y(\cdot))}^{q}\right)
$$

and

$$
y \in K N_{c}(y) \quad\left(N_{c}(y)=S_{\overline{\operatorname{conv}} F(\cdot, y(\cdot))}^{q}\right) .
$$

In what follows, by $S \subseteq L^{p}(Z, X)$ we denote the solution set of (6) and by $S_{c} \subseteq L^{p}(Z, X)$ the solution set of (7). Also here in order to simplify our calculations, we shall assume that $\mu(Z)=1$.

$\mathbf{H}(\mathbf{F})_{5} . F: Z \times X \rightarrow P_{f}\left(X^{*}\right)$ is a multifunction such that (i) for every $y \in X, z \rightarrow$ $F(z, y)$ is measurable; (ii) there exists $l \in L^{\infty}(Z)$ such that $h\left(F(z, y), F\left(z, y^{\prime}\right)\right) \leq$ $l(z)\left\|y-y^{\prime}\right\| \mu$-a.e. on $Z$ for all $y, y^{\prime} \in X$; (iii) for almost all $z \in Z$ and all $y \in X$, $|F(z, y)| \leq a(z)+c\|y\|^{p-1}$ with $a \in L^{q}(Z), c \geq 0$; (iv) hypothesis $H(F)_{2}$ (iv) holds. 
Theorem 8. If hypotheses $H(F)_{5}, H(K)_{1}$ hold, $\|K\|_{\mathcal{L}}<1$, $\|l\|_{\infty}<1$ and $p \geq 2$,

then $S_{c}=\bar{S}$ where the closure taken in $L^{p}(Z, X)$.

Proof. Let $y \in S_{c}$. Then $y=K u$ with $u \in N_{c}(y)$. Given $\varepsilon>0$ we can find $U$ a symmetric weak neighborhood of the origin in $L^{q}\left(Z, X^{*}\right)$ such that $u-u_{1} \in U$ implies $\left\|y-y_{1}\right\|_{p}=\left\|K u-K u_{1}\right\|_{p}<\varepsilon\left(y_{1}=K u_{1}\right)$. By virtue of Proposition 4.1 of Papageorgiou [17], we can have $u_{1} \in N(y)=S_{F(\cdot, y(\cdot))}^{q}$. Via an easy application of Aumann's selection theorem, we can produce $u_{2} \in N\left(y_{1}\right)$ such that $\| u_{1}(z)-$ $u_{2}(z)\left\|=h\left(F(z, y(z)), F\left(z, y_{1}(z)\right)\right) \leq l(z)\right\| y(z)-y_{1}(z) \| \mu$-a.e. on $Z$ and so $\| u_{1}-$ $u_{2}\left\|_{q} \leq\right\| l\left\|_{\infty}\right\| y-y_{1} \|_{p}($ recall $\mu(Z)=1$ and $p \geq 2)$. Suppose $u_{1}, \ldots, u_{n} \in L^{q}\left(Z, X^{*}\right)$ have been chosen such that $\left\|u_{k+1}-u_{k}\right\|_{q} \leq\|l\|_{\infty}^{k} \varepsilon, u_{k+1} \in N\left(y_{k}\right), y_{k}=K u_{k}$, $k=1,2, \ldots, n-1$. We define $y_{n}=K u_{n}$. For $k=1,2, \ldots, n-1$, we have $\left\|y_{k+1}-y_{k}\right\|_{p}=\left\|K u_{k+1}-K u_{k}\right\|_{p} \leq\left\|u_{k+1}-u_{k}\right\|_{q} \leq\|l\|_{\infty}^{k} \varepsilon$. Hence

$$
\left\|y_{k+1}-y\right\|_{p} \leq \sum_{r=0}^{k}\left\|y_{r+1}-y_{r}\right\|_{p} \leq \varepsilon \sum_{r=0}^{k}\|l\|_{\infty}^{r} .
$$

A new application of Aumann's selection theorem gives us $u_{n+1} \in N\left(y_{n}\right)$ such that $\left\|u_{n+1}(z)-u_{n}(z)\right\|=h\left(F\left(z, y_{n}(z)\right), F\left(z, y_{n-1}(z)\right)\right) \leq l(z)\left\|y_{n}(z)-y_{n-1}(z)\right\| \mu$-a.e. on $Z$. Hence $\left\|u_{n+1}-u_{n}\right\|_{q} \leq\|l\|_{\infty}\left\|y_{n}-y_{n-1}\right\|_{p} \leq\|l\|_{\infty}^{n} \varepsilon$. Therefore by induction we have produced a sequence $\left\{u_{n}\right\}_{n \geq 1} \subseteq L^{q}\left(Z, X^{*}\right)$ such that $\left\|u_{n+1}-u_{n}\right\|_{q} \leq$ $\|l\|_{\infty}^{n} \varepsilon, n \geq 1$. Since $\|l\|_{\infty}<1$, we see that $\left\{u_{n}\right\}_{n \geq 1}$ is Cauchy in $L^{q}\left(Z, X^{*}\right)$. So $u_{n} \rightarrow \hat{u}$ in $L^{q}\left(Z, X^{*}\right)$. Then $y_{n}=K u_{n} \rightarrow K \hat{u}=\hat{y}$ in $L^{p}(Z, X)$. Evidently by virtue of hypothesis $H(F)_{5}$ (ii) $\hat{u} \in N(\hat{y})$ and so $\hat{y} \in S$. Moreover $\left\|y_{n}-y\right\|_{p} \leq$ $\varepsilon \sum_{k=0}^{n-1}\left\|y_{k+1}-y_{k}\right\|_{p} \leq \varepsilon \sum_{k=0}^{n-1}\|l\|_{\infty}^{k}\left(y_{0}=y\right)$. Hence in the limit as $n \rightarrow \infty$ we have $\|\hat{y}-y\|_{\infty} \leq \varepsilon \frac{1}{1-\|l\|_{\infty}}$. Since $\varepsilon>0$ was arbitrary and $S_{c}$ is closed in $L^{p}(Z, X)$, we conclude that $S_{c}=\bar{S}$, the closure in $L^{p}(Z, X)$.

\section{EXAMPLES}

In this section we outline some examples which illustrate the applicability of our results.

(a) "Elliptic inclusions": Let $Z \subseteq \mathbb{R}^{N}, N \geq 1$, be a bounded domain with a $C^{1}$ boundary $\Gamma$. Let $L$ be a uniformly elliptic linear differential operator of order $2 m$ in divergence form; i.e. $L=\sum_{|\alpha|,|\beta| \leq m}(-1)^{|\alpha|} D^{\alpha}\left(a_{\alpha \beta}(z) D^{\beta}\right)$. Here $z=(z)_{k=1}^{N}, D_{k}=$ $\frac{\partial}{\partial_{z_{k}}}, \alpha=\left(\alpha_{1}, \ldots, \alpha_{N}\right)$ is a multi-index (i.e. an $N$-tuple of nonnegative integers), $|\alpha|=\sum_{k=1}^{N} \alpha_{k}$ (the length of the multi-index) and $D^{\alpha} u=D_{1}^{\alpha_{1}} \cdots D_{N}^{\alpha_{N}} u$. For $\alpha=0$, we set $D^{0} u=u$. We shall make the following hypotheses:

$\mathbf{H}(\mathbf{a}) . a_{\alpha \beta}=a_{\beta \alpha} \in L^{\infty}\left(Z, \mathbb{R}^{N} \times \mathbb{R}^{N}\right)$ and there exists $\eta>0$ such that for all $v=$ $\left(v_{\alpha}\right)_{|\alpha| \leq m} \in \mathbb{R}^{N_{m}}\left(N_{m}=\frac{(N+m) !}{m ! N !}\right)$, we have $\sum_{|\alpha|,|\beta| \leq m}\left(a_{\alpha \beta}(z) v_{\alpha}, v_{\beta}\right)_{\mathbb{R}^{N}} \geq \eta\|v\|_{\mathbb{R}^{N_{m}}}^{2}$ a.e. on $Z$.

We consider the following Dirichlet multivalued elliptic system:

$$
\left\{\begin{array}{l}
(L y)(z) \in F(z, y(z)) \text { a.e. on } Z \\
\left.D^{\gamma} y\right|_{\Gamma}=0,|\gamma| \leq m-1
\end{array}\right\} \text {. }
$$

We shall need the following hypotheses on the multifunction $F$ :

$\mathbf{H}(\mathbf{F})_{6} . F: Z \times \mathbb{R}^{N} \rightarrow P_{f c}\left(\mathbb{R}^{N}\right)$ is a multifunction such that (i) for every $y \in \mathbb{R}^{N}$, $z \rightarrow F(z, y)$ is measurable; (ii) for almost all $z \in Z, \operatorname{GrF}(z, \cdot)$ is closed; (iii) for 
almost all $z \in Z$ and all $y \in \mathbb{R}^{N},|F(z, y)| \leq a(z)+c\|y\|^{p-1}$ with $a \in L^{2}(Z), c \geq 0$, $p \geq 2$; (iv) there exist $\theta \in L^{1}(Z)$ and $c_{0}>0$ such that for almost all $z \in Z$, all $y \in \mathbb{R}^{N}$ and all $u \in F(z, y),(-u, y)_{\mathbb{R}^{N}} \geq c_{0}\|y\|^{p}-\theta(z)$.

Let $X=W_{0}^{m, p}\left(Z, \mathbb{R}^{N}\right), H=L^{2}\left(Z, \mathbb{R}^{N}\right)$ and $X^{*}=W^{-m, q}\left(Z, \mathbb{R}^{N}\right)$. This is an evolution triple with the embeddings $X \hookrightarrow H \hookrightarrow X^{*}$ being compact. Let $\widehat{L}: X \rightarrow X^{*}$ be defined by

$$
\langle\widehat{L}(y), u\rangle=\int_{Z} \sum_{|\alpha|,|\beta| \leq m}\left(a_{\alpha \beta}(z) D^{\beta} y(z), D^{\alpha} u(z)\right)_{\mathbb{R}^{N}} d z \quad \text { for all } y, u \in X
$$

Here $\langle\cdot, \cdot\rangle$ denotes the duality brackets for the pair $\left(X, X^{*}\right)$. Note that $\widehat{L}$ is a continuous linear operator such that $\|\widehat{L} y\|_{*} \geq \eta\|y\|$ for all $y \in X$. Let $K: H \rightarrow$ $X \hookrightarrow H$ be the abstract Green's operator corresponding to $\widehat{L}$. It is well-known that $K$ is compact, linear, monotone. Let $N: H \rightarrow P_{f c}(H)$ be the multivalued Nemitsky operator corresponding to $F$. Then problem (8) is equivalent to the Hammerstein integral inclusion $y \in K N(y)$, which by Theorem 5 has a solution.

Remark. This example extends the result of Marano [12], where $N=1$ and so $F(z, y)=\left[f_{1}(z, y), f_{2}(z, y)\right]$ with $f_{1}(z, \cdot)$ lower semicontinuous and $f_{2}(z, \cdot)$ upper semicontinuous. Such problems arise when we deal with elliptic equations with discontinuous nonlinearities (see Chang [7]). The hypotheses of Marano are more restrictive and his approach different from ours.

(b) "Control systems with a priori feedback": Let $L$ be the same linear differential operator as in example (a). We consider the following elliptic control system with a priori feedback:

$$
\left\{\begin{array}{l}
(L y)(z)=f(z, y(z), u(z)) \text { a.e. on } Z \\
\left.D^{\gamma} y\right|_{\Gamma}=0,|\gamma| \leq m-1, u(z) \in U(z, y(z)) \text { a.e. on } Z
\end{array}\right\} .
$$

We shall need the following hypotheses on the data of (9):

$\mathbf{H}(\mathbf{F}) . f: Z \times \mathbb{R}^{N} \times \mathbb{R}^{k} \rightarrow \mathbb{R}^{N}$ is a Caratheodory function (i.e. measurable in $z \in Z$ and continuous in $\left.(y, u) \in \mathbb{R}^{N} \times \mathbb{R}^{k}\right)$ such that (i) there exist $a \in L^{q}(Z)$ and $c \geq 0$ such that for almost all $z \in Z$ and all $(y, u) \in \mathbb{R}^{N} \times \mathbb{R}^{k},\|f(z, y, u)\| \leq$ $a(z)+\bar{c}\left(\|y\|^{p-1}+\|u\|^{p-1}\right)$; (ii) there exist $\theta \in L^{1}(Z)$ and $c_{0}>0$ such that for almost all $z \in Z$, all $y \in \mathbb{R}^{N}$ and all $u \in U(z, y)$ we have $(-f(z, y, u), y)_{\mathbb{R}^{N}} \geq c_{0}\|y\|^{p}-\theta(z)$.

$\mathbf{H}(\mathbf{U}) . U: Z \times \mathbb{R}^{N} \rightarrow P_{k}\left(\mathbb{R}^{k}\right)$ is measurable in $z \in Z, h$-continuous in $y$ and there exist $a_{1} \in L^{2}(Z)$ and $c_{1} \geq 0$ such that for almost all $z \in Z$ and all $y \in \mathbb{R}^{N}$, $|U(z, y)| \leq a_{1}(z)+c_{1}\|y\|$.

Let $F: Z \times \mathbb{R}^{N} \rightarrow P_{k}\left(\mathbb{R}^{N}\right)$ be defined by $F(z, y)=f(z, y, U(z, y))$. Let $y \in$ $L^{2}(Z)$. Then by virtue of $H(U), z \rightarrow U(z, y(z))$ is measurable (see [16]). Thus we can find $u_{n}: Z \rightarrow \mathbb{R}^{k}, n \geq 1$, measurable functions such that $U(z, y(z))=$

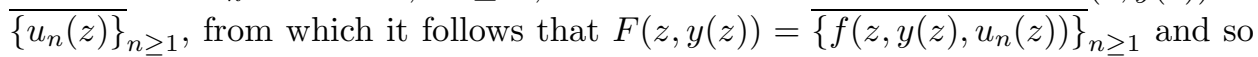
$F(\cdot, \cdot)$ is superpositionally measurable. Also we claim that $F(z, \cdot)$ is lsc. Indeed let $y_{n} \rightarrow y$ in $\mathbb{R}^{N}$ and let $v \in F(z, y)$. Then $v=f(z, y, u)$. Let $u_{n} \in U\left(z, y_{n}\right)$, $n \geq 1$, such that $u_{n} \rightarrow u$ in $\mathbb{R}^{k}$. Then $v_{n}=f\left(z, y_{n}, u_{n}\right) \in F\left(z, y_{n}\right)$ and $v_{n} \rightarrow v$. So $F(z, y) \subseteq \underline{\lim } F\left(z, y_{n}\right)$ and this means that $F(z, \cdot)$ is lsc (see Klein-Thompson [10]). We continue as in example (a), using this time Theorem 7 ("nonconvex" problem). 
Remark. An alternative set of hypotheses for $U(t, y)$ is the following: $z \rightarrow U(z, y)$ is measurable, $y \rightarrow U(z, y)$ is lsc, $U(\cdot, \cdot)$ is separable (see [16]) and $|U(z, y)| \leq$ $a_{1}(z)+c_{1}\|y\|$ a.e. on $Z, a_{1} \in L^{1}(Z), c_{1} \geq 0$.

(c) "Random integral inclusions": Let $(\Omega, \Sigma, P)$ be a complete probability space and $Z \subseteq \mathbb{R}^{N}$ a bounded domain, $N \geq 1$. We consider the following random integral inclusion:

$$
y(z, \omega) \in \int_{Z} k(z, \tau, \omega) F(\tau, \omega, y(\tau, \omega)) d \tau .
$$

$\mathbf{H}(\mathbf{K})_{3} . \quad K: Z \times Z \times \Omega \rightarrow \mathcal{L}\left(\mathbb{R}^{N}, \mathbb{R}^{N}\right)$ is measurable and (i) for each $u \in L^{q}\left(\Omega, \mathbb{R}^{N}\right)$, $k(z, \tau, \cdot) u(\cdot) \in L^{p}\left(\Omega, \mathbb{R}^{N}\right)$ for all $z, \tau \in Z$ (i.e. $\hat{k}(z, \tau)=k(z, \tau, \cdot)(\cdot) \in \mathcal{L}\left(L^{q}\left(\Omega, \mathbb{R}^{N}\right)\right.$, $\left.\left.L^{p}\left(\Omega, \mathbb{R}^{N}\right)\right)\right)$, where $1<p<\infty, \frac{1}{p}+\frac{1}{q}=1$; (ii) $k \in L^{p}\left(Z \times Z \times \Omega, \mathcal{L}\left(\mathbb{R}^{N}, \mathbb{R}^{N}\right)\right)$; (iii) there exist $\beta_{1}>0$ and $1<\gamma<q$ such that for almost all $(z, \tau, \omega) \in Z \times Z \times \Omega$ and all $u \in \mathbb{R}^{N}$, we have $(k(z, \tau, \omega) u, u)_{\mathbb{R}^{N}} \geq-\beta_{1}\|u\|^{\gamma}$.

$\mathbf{H}(\mathbf{F})_{\mathbf{7}} . F: Z \times \Omega \times \mathbb{R}^{N} \rightarrow P_{k c}\left(\mathbb{R}^{N}\right)$ is a multifunction such that (i) for all $y \in \mathbb{R}^{N}$, $(z, \omega) \rightarrow F(z, \omega, y)$ is measurable; (ii) for all $(z, \omega) \in Z \times \Omega, y \rightarrow F(z, \omega, y)$ is $h$-continuous; (iii) there exist $a \in L^{q}(Z \times \Omega)$ and $c \in L^{\infty}(\Omega)$ such that for almost all $(z, \omega) \in Z \times \Omega$ and all $y \in \mathbb{R}^{N},|F(z, \omega, y)| \leq a(z, \omega)+c(\omega)\|y\|^{p-1}$; (iv) there exist $\theta \in L^{1}(Z \times \Omega)$ and $c_{0} \in L^{\infty}(\Omega)$ such that for almost all $(z, \omega) \in Z \times \Omega$ and all $y \in \mathbb{R}^{N},(-u, y)_{\mathbb{R}^{N}} \geq c_{0}\|y\|^{p}-\theta(z, \omega)$.

For each $z \in Z$ and $y \in L^{p}\left(\Omega, \mathbb{R}^{N}\right)$, let $\widehat{F}(z, y)=S_{F(z, \cdot, y(\cdot))}^{q}$. Then it is easy to check that $\widehat{F}: Z \times L^{p}\left(\Omega, \mathbb{R}^{N}\right) \rightarrow P_{f c}\left(L^{q}\left(\Omega, \mathbb{R}^{N}\right)\right)$ satisfies hypothesis $H(F)_{3}$. Moreover if $K: L^{q}\left(Z, L^{q}\left(\Omega, \mathbb{R}^{N}\right)\right) \rightarrow L^{p}\left(Z, L^{p}\left(\Omega, \mathbb{R}^{N}\right)\right)$ is defined by $K(u)(z)=$ $\int_{Z} k(z, \tau, \cdot) u(\tau, \cdot) d \tau$ (Bochner integral), then recalling that $L^{r}\left(Z, L^{r}\left(\Omega, \mathbb{R}^{N}\right)\right)=$ $L^{r}\left(Z \times \Omega, \mathbb{R}^{N}\right), 1 \leq r$, we see that $K$ satisfies $H(K)_{1}$. So we can apply Theorem 5 and obtain a $y \in L^{p}\left(Z \times \Omega, \mathbb{R}^{N}\right)$ which solves $(10)$.

(d) "Evolution inclusions": Let $T=[0, b]$ and $H$ be a separable Hilbert space. Let $\{A(t)\}_{t \in T}$ be a family of densely defined linear operators in $H$, which generates an evolution operator $\{U(t, s): 0 \leq s \leq t \leq b\}$ on $H$, such that $U(t, s)$ is compact for $s<t$. We consider the following Cauchy problem:

$$
\left\{\begin{array}{l}
\dot{y}(t) \in A(t) y(t)+F(t, y(t)) \text { on } T \\
y(0)=y_{0}
\end{array}\right\} .
$$

By a solution of (11) we mean a mild solution, i.e. a function $y \in C(T, H)$ such that $y(t)=U(t, 0) y_{0}+\int_{0}^{t} U(t, s) u(s) d s$ for all $t \in T$, with $u \in N(y)=S_{F(\cdot, y(\cdot))}^{p}$. If $F$ satisfies $H(F)_{2}$ or $H(F)_{3}$ and for all $u \in L^{q}(T, H), \int_{0}^{b}\left(\int_{0}^{t} U(t, s) u(s) d s, u(t)\right)_{H} d t \geq$ 0 , then we can apply Theorem 4 or Theorem 5 and produce a mild solution for problem (11).

(e) "Nonsmooth energy functionals": Let $X=W_{0}^{m, p}\left(Z, \mathbb{R}^{N}\right)$, and let $X^{*}=$ $W^{-m, q}\left(Z, \mathbb{R}^{N}\right)$. Let $L$ be the uniformly elliptic operator of example (a) and let $\widehat{L}: X \rightarrow X^{*}$ be the corresponding linear operator. Assume that $f: Z \times \mathbb{R}^{N} \rightarrow \mathbb{R}$ is a Caratheodory function, which is convex in the variable $y \in \mathbb{R}^{N}$. Consider the convex functional $\varphi(y)=\frac{1}{2}\langle\widehat{L} y, y\rangle-V(y), y \in X$, where $V(y)=\int_{Z} f(z, y(z)) d z$. We are looking for critical points of $\varphi(\cdot)$; i.e. for $y \in X$ such that $0 \in \partial \varphi(y)$, where $\partial \varphi(y)$ denotes the subdifferential of $\varphi$ at $y$. Assume that for every $y \in L^{p}\left(Z, \mathbb{R}^{N}\right)$, $V(y)$ is finite. Then by Theorem 2.2 of Chang [7] we know that $\partial V(Y)=N_{\partial f}(y)=$ $S_{\partial f}^{q}(y) \subseteq L^{q}\left(Z, \mathbb{R}^{N}\right)$ for all $y \in X$. Also assume that for almost all $z \in Z$ and all 
$y \in \mathbb{R}^{N},-f^{\prime}(z, y ; y) \geq c_{0}\|y\|^{p}-\theta(z)$, where $\theta \in L^{1}(Z), c_{0}>0$ (here $f^{\prime}(z, y ; h)$ denotes the directional derivative of $f(z, \cdot)$ at $y$ in the direction $h)$. Then finding critical points of $\varphi(\cdot)$ is equivalent to solving the inclusion $\widehat{L} y \in \partial V(y)$, which, by virtue of our hypothesis, can be solved by invoking Theorem 4 .

\section{REFERENCES}

[1] N. U. Ahmed, Nonlinear integral equations on reflexive Banach spaces with applications to stochastic integral equations and abstract evolution equations, J. Integral Eqns. 1 (1979), pp. $1-15$.

[2] H. Amann, Existence theorems for equations of Hammerstein type, Appl. Anal. 1 (1972), pp. 385-397. MR 52:15142

[3] J. Appell, E. De Pascale, H. T. Hguyen and P. Zabrejko, Nonlinear integral inclusions of Hammerstein type, Top. Meth. Nonl. Anal. 5 (1995), pp. 111-124. MR 97f:45003

[4] A. Bressan and G. Colombo, Extensions and selections of maps with decomposable values, Studia Math. 90 (1988), pp. 69-85. MR 89j:54021

[5] H. Brezis and F. Browder, Existence theorems for nonlinear integral equations of Hammerstein and Urysohn type, Bull. AMS 81 (19750, pp. 73-78. MR 53:11441

[6] F. Browder, Nonlinear functional analysis and nonlinear integral equations of Hammerstein and Urysohn type in Contributions to Nonlinear Functional Analysis, ed. E. Zarantonello, Academic Press, New York (1971), pp. 425-500. MR 52:15143

[7] K. C. Chang, Variational methods for nondifferentiable functionals and their applications to partial differential equations, J. Math. Anal. App. 80 (1981), pp. 102-129.

[8] C. V. Coffmann, Variational theory of set-valued Hammerstein operators in Banach spaces. The eigenvalue problem, Ann. Scuola Norm. Sup. Pisa 4 (1978), pp. 633-655.

[9] K. Glashoff and J. Sprekels, An application of Glicksberg's theorem to set-valued integral equations arising in the theory of thermostats, SIAM J. Math. Anal. 12 (1981), pp. 477-486. MR 82f: 45008

[10] E. Klein and A. Thompson, Theory of Correspondences, Wiley, New York, (1984).

[11] L. Lyapin, Hammerstein inclusions, Diff. Equations 10 (1976), pp. 638-643.

[12] S. Marano, Existence theorems for a semilinear elliptic boundary value problem, Annales Polon. Math. LX (1994), pp. 57-67. MR 95e:35076

[13] D. O'Regan, Integral equations in reflexive Banach spaces and weak topologies, Proc. AMS 124 (1996), pp. 607-614. MR 96d:45003

[14] Integral inclusions of upper semicontinuous or lower semicontinuous type, Proc. AMS 124 (1996), pp. 2391-2399. MR 96j:47064

[15] N. S. Papageorgiou, Convergence theorems for Banach spaces valued integrable multifunctions, Intern. J. Math and Math. Sci. 10 (1987), pp. 433-442. MR 88i:28019

[16] - On measurable multifunctions with applications to random multivalued equations, Math. Japonica 32 (1987), pp. 437-464. MR 89a:54019

[17] _ Measurable multifunctions and their applications to convex integral functionals, Intern. J. Math. Sci. 12 (1989), pp. 175-192. MR 90f:49009

[18] _ Existence of solutions for integral inclusions of Urysohn type with nonconvex valued orientor field, Jour. Optim. Th. Appl. 64 (1990), pp. 207-215. MR 91i:47079

Department of Mathematics, University of Perugia, Via Vanvitelli 1, Perugia 06123, ITALY

Department of Mathematics, National Technical University, Zografou Campus, Athens 157 80, Greece

E-mail address: npapg@math.ntua.gr 